\title{
Deblocking of Interacting Particle Assemblies: from Pinning to Jamming
}

\author{
M.-Carmen Miguel, \\ Departament de Física Fonamental, Facultat de Física, Universitat de Barcelona, Av. Diagonal 647, E-08028, Barcelona, Spain
}

José S. Andrade Jr., Departamento de Física, Universidade Federal do Ceará, 60451-970 Fortaleza, Ceará, Brazil.

and Stefano Zapperi

INFM unità di Roma 1 and SMC, Dipartimento di Fisica, Università “La Sapienza”, P.le A. Moro 2, 00185 Roma, Italy

Received on 24 April, 2003

\begin{abstract}
A wide variety of interacting particle assemblies driven by an external force are characterized by a transition between a blocked and a moving phase. The origin of this deblocking transition can be traced back to the presence of either external quenched disorder, or of internal constraints. The first case belongs to the realm of the depinning transition, which, for example, is relevant for flux-lines in type II superconductors and other elastic systems moving in a random medium. The second case is usually included within the so-called jamming scenario observed, for instance, in many glassy materials as well as in plastically deforming crystals. Here we review some aspects of the rich phenomenology observed in interacting particle models. In particular, we discuss front depinning, observed when particles are injected inside a random medium from the boundary, elastic and plastic depinning in particle assemblies driven by external forces, and the rheology of systems close to the jamming transition. We emphasize similarities and differences in these phenomena.
\end{abstract}

\section{Introduction}

Various materials ranging from synthetic nanocrystals, magnetic colloids, charged particles in Coulomb crystals, proteins and surfactants, or vortices in type II superconductors and in Bose-Einstein condensates, form ordered selfassembled structures. This topic has attracted much interest for various fundamental and technological reasons. In this respect, the response of these structures to external forces of various kinds (optical, magnetic, mechanical) is of particular importance [1-3]. In many cases one observes the presence of blocked phases, where the evolution of the system is frozen. This behavior can have different origins: when it is due to the presence of quenched disorder it is denoted by pinning, while when it is due to intrinsic constraints it is referred to as "jamming". In both cases, a sufficiently large force leads to a moving phase, through a deblocking transition.

All these systems can be modeled by a set of interacting particles moving under the action of external forces sometimes in a random pinning field. For instance, superconducting vortices in thin films are pinned by vacancies and driven by an applied current through the Lorentz force, colloids interact replace by via Van der Waals or dipolar forces and are driven by the solvent flow. Despite the differences in these systems, one can try to identify some common features in their dynamic response. This goal has been achieved mostly through the use of numerical simulations, which have been extensively employed in the past in various contexts. Here we review the results obtained from numerical simulations of interacting particles, in order to provide a common framework for pinning and jamming phenomena that, despite their similarities, have been traditionally studied by different communities.

The transition from a blocked to a moving phase is a central problem in the theory of non-equilibrium critical phenomena. Beside the large body of theoretical work devoted to the depinning transition of elastic manifolds in disordered media and recent theories devoted to jamming in glasses and colloids, one should also mention the theory of absorbingstate phase transitions $[4,5]$. An absorbing state is a configuration in which the evolution of the system, typically a stochastic lattice model, is frozen and no longer evolves. When a suitable control parameter is changed the system can eventually be found into an active, statistically stationary, phase. The absorbing state phase transition is a second order non-equilibrium phase transition, characterized by scaling laws and critical exponents, as in ordinary equilibrium phase transitions. The same is true for the depinning transition and, in fact, it is sometimes possible to map a depinning transition into and absorbing-state phase transition and vice versa. While the characterization of pinning-depinning as a critical phenomenon is based on a firm theoretical ground, the current theoretical understanding of jamming phenom- 
ena is not so advanced. Similarly, when depinning involves the generation of topological defects, one refers to a plastic depinning transition, but the precise meaning of the word transition is not clear at present in this context.

At this stage of the theoretical understanding, however, it is possible to draw an extensive common picture of these phenomena, in which some parts are depicted in full detail, others are less precise, and some are just sketched. We hope that this work, by its own nature incomplete, will stimulate others to find further connections between non-equilibrium transitions from blocked to moving phases and possibly to formulate a complete theory encompassing all these phenomena.

The paper is organized as follows: in section II we discuss the models used to describe the physics of interacting particle assemblies. In section III, we analyze the injection of particles in a random medium and discuss the relations with front propagation and with continuum theories. Section IV is devoted to the depinning of interacting particles by an external force through a pinning field. We analyze the problem by increasing gradually the level of complexity, from the pinning of single particle to collective elastic and plastic depinning. In section $\mathrm{V}$, we introduce jamming phenomena and discuss in detail the jamming transition observed in plastically deformed crystals, modeled by a set of stress-driven interacting dislocations. We conclude briefly in section VI.

\section{Interacting particle models and their physical realization}

Several systems in nature can be modeled by a collection of interacting particles. Here we summarize the main features of these models and discuss some concrete examples. For simplicity, we will restrict ourselves to pairwise interactions between particles. In this case, identifying the particle coordinates by $\vec{r}_{i}$, with $i=1, \ldots N$, we can write the equations of motion in general as

$$
m \frac{d^{2} \vec{r}_{i}}{d t^{2}}+\Gamma \frac{d \vec{r}_{i}}{d t}=\sum_{j} \vec{J}\left(\vec{r}_{j}-\vec{r}_{i}\right)+\vec{F}_{e x t}\left(\vec{r}_{i}, t\right),
$$

where $m$ is the mass of the particles, $\Gamma$ is a damping coefficient, and $\vec{J}=-\vec{\nabla} V(\vec{r})$ is the interparticle force derived from an interaction potential $V$. The last term represents external forces, quenched disorder, or other noise sources, and will be discussed in detail below. In most cases of interest, dissipation is so strong that we can safely neglect inertia putting $m=0$. Most of the following discussion will be devoted to this overdamped limit, but occasionally we will discuss as well the effect of inertia.

Depending of the particular system under study, the interparticle potential can have different forms which will affect the dynamics of the system. The simplest case is that of a short-range repulsive central force $\vec{J}(\vec{r})=\hat{r} K\left(|\vec{r}| / \xi_{v}\right)$ which can be characterized by its peak value $K(0)$ and its range $\xi_{v}$. In a series of increasing complexity, one can consider non-monotonic interactions (i.e. the force can be re- pulsive and attractive in different ranges), long-range interactions (i.e. $K(r) \sim r^{-\alpha}$, for large $r$ with $0<\alpha<d+2$, anisotropic forces (i.e. $\vec{J}=\hat{r} K(\vec{r})$ ), non-central forces (i.e. $\vec{J}(\vec{r}) \times \hat{r} \neq 0$ ), or different combinations of the above.

The external force normally includes a uniform driving force $F$, which could be time dependent. Typical examples are the AC drive $F(t)=F_{0} \sin (\omega t)$ or the ramp up $F(t)=c t$, but the possibilities are endless. In addition, one should consider position dependent forces due to quenched impurities that may be present in the system. Here we will mainly discuss the effect of a set of $N_{p}$ pinning points placed randomly at position $\vec{R}_{p}$, giving rise to a random force field of the type

$$
F_{p}(\vec{r})=\sum_{p} \vec{f}_{p}\left(\left(\vec{r}-\vec{R}_{p}\right) / \xi_{p}\right)
$$

where $\xi_{p}$ is the range of the individual pinning forces. Normally, the particular shape of the pinning potential does not matter as long as its range is short. One can also consider correlated disorder, such as columnar and planar defects, depending on the particular situation at hand. In addition, thermal effects can be included adding a a random uncorrelated Gaussian term $\eta(\vec{r}, t)$ to the equation.

Once the interactions of the particle systems have been specified, one should also discuss the boundary and initial conditions of the model. A common choice is to use periodic boundary conditions, and to place the particles in their zero temperature equilibrium positions (i.e. forming a crystal). Alternatively, the particles can be placed randomly in the system mimicking a sudden quench from a disordered high temperature phase. The latter may give rise to an intrinsic geometrical disorder. These conditions are appropriate if one is interested in modeling the dynamics in the bulk of the material, without worrying about surface effects. On the other hand, boundary effects are at the core of the phenomena in some cases and one should then implement different initial and boundary conditions. This case will be discussed explicitly in the next section. Periodic boundary conditions need special care when interactions are long-ranged, since in this case one cannot impose a cutoff to the extent of the interaction force, as it is often done for short range-forces. One should instead consider explicitly the interaction of the particles in a given finite cell with all the periodic images of the system. The infinite sum over the images can rarely be performed exactly and since the sum is slowly converging a simple truncation of the series gives a poor approximation and may induce spurious effects. To overcome this problem one can employ the Ewald summation method, originally proposed for Coulomb interactions, after generalizing it for the appropriate interactions involved [6].

Finally, we would like to discuss here some physical realizations of the generic model we have discussed above. One of the most studied examples is the flux-line lattice in type II superconductors. In thin superconducting films, the system can be really treated as a quasi-two dimensional set of interacting rod-like particles. Conversely, for thicker superconductor samples one should study a set of flexible lines. In this article, we will only consider the case of rigid 
vortex lines. The interparticle force between rigid flux-lines in the framework of the London theory is given by

$$
\vec{J}(\vec{r})=\Phi_{0}^{2} /\left(8 \pi^{2} \lambda^{3}\right) K_{1}(|\vec{r}| / \lambda) \hat{r}
$$

where $\Phi_{0}$ is the quantized flux carried by the vortices, $K_{1}$ is a Bessel function and $\lambda$ is the London penetration length $[28,27]$. Notice that this is a short-range (since $K_{1}(x) \sim$ $\exp (-x)$ for large $x)$ repulsive central force, with a divergence of the form $x^{-1}$ at short distances which is cut off by the vortex core. Instead in two dimensions, the interaction is long-range

$$
\vec{J}(|\vec{r}|)=\frac{\Phi_{0}^{2} \hat{r}}{8 \pi^{2} \lambda^{2} r}
$$

decaying as $1 / r$. In addition to interaction forces, a current $\vec{j}$ flowing in the superconductor produces a Lorentz force $\vec{F}=\vec{j} \times \vec{B} / c$ acting on the vortices.

In the case of complex fluids or soft condensed matter materials [7], which usually contain large polymer molecules or colloidal particles in a solvent whose molecules are much smaller in size, a generic model in which inertial terms are neglected often provides an effective approach towards describing such systems. The solvent is considered as a continuum medium, characterized by its viscosity, in which energy is dissipated as the suspended particles move through it. In close correspondence with their characteristic dissipative motion, the suspended particles exhibit a Brownian dynamics due to the random collisions with the solvent molecules. This is modeled as a random Gaussian force $\vec{\eta}$ in the equations of motion of the form

$$
\Gamma_{i}\left(\frac{d \vec{r}_{i}}{d t}-\vec{v}^{s}\right)=\vec{J}\left(\vec{r}_{i}\right)+\vec{\eta}\left(\vec{r}_{i}, t\right),
$$

where $\vec{v}^{s}$ is the solvent velocity that can be controlled by an externally applied flow field, and $\vec{J}$ is an elastic or conservative force on particle $i$ due to deformations of long molecules or bubbles, or due to other interactions (such as Van der Waals, electrostatic, magnetic, and excludedvolume) among the suspended particles. The amplitude of the autocorrelation function $\left\langle\left|\vec{\eta}_{i}\right|^{2}\right\rangle=2 \Gamma_{i} k_{B} T \delta\left(t-t^{\prime}\right)$ is proportional to the temperature $T$ of the system. More sophisticated algorithms, in which one solves similar equations to the one represented above, have been developed to model the rheology of dense spherical particles [8] accounting for hydrodynamic interactions, ellipsoidal or rod-like particles [9], as well as emulsions [10] and foams [11].

Another example which is worth considering from this general point of view is a collection of dislocations in a thin crystalline film. Crystal dislocations are topological defects characterized by a Burgers vector $\vec{b}$ [12]. As in the case of flux-lines, in a three dimensional crystal dislocations are deformable lines. Nevertheless, one often treats them in the rigid approximation, obtaining an effective two dimensional particle model, which becomes exact for thin samples. Dislocations produce long-range stress and strain fields in the host crystal, and experience the so-called Peach-Koehler force due to the overall local stress. This induces an interaction force between dislocations that depends on their character (edge or screw, when $\vec{b}$ is perpendicular or parallel to the corresponding dislocation axis, respectively [12]), but that is generally long-range, decaying as $1 / r$, and anisotropic. For instance, the force between two edge dislocations at a distance $\vec{r}=(x, y)$, and with Burgers vectors in the $x$ direction is given by

$$
J_{x}(x, y)=\frac{\mu b^{2}}{2 \pi(1-\nu)} \frac{x\left(x^{2}-y^{2}\right)}{\left(x^{2}+y^{2}\right)^{2}},
$$

where $\mu$ is the shear modulus, and $\nu$ is the Poisson ratio of the host crystal. We have only considered the $x$ component since, differently from flux lines, dislocations move mainly by gliding along preferential directions, namely the direction of the Burgers vector. Thus, while the particle system is two dimensional, the motion is confined along several particular directions. This fact, together with the anisotropic character of the interaction, gives rise to metastable structures that act as geometric constrains for their own dynamics. In this case, the driving force is often an externally applied stress $\sigma$ which acts on the dislocations through the Peach-Koehler force $\vec{F}=(\vec{b} \cdot \sigma) \times \hat{L}$, where $\hat{L}$ is the direction of the dislocation line local tangent.

\section{Gradient driven dynamics: front invasion}

The theoretical and experimental investigation of the growth dynamics of rough interfaces has became a subject of great scientific interest in recent years [13-15]. This is clearly illustrated nowadays by the large variety of studies dealing with front invasion where roughening processes take place such as flow through porous media [16-18] or imbibition [19], flame propagation [20, 21], deposition processes $[14,15]$, and flux penetration in superconducting materials $[32,33,45,46]$.

From a macroscopic point of view, the development of modeling techniques for the description of these dynamical systems has been generally based on the traditional approach to transport phenomena, where the governing expressions are usually differential equations representing local balances of the quantity of interest (e.g., mass, momentum, flux of superconducting vortices, etc.) in a continuum framework. However, it is sometimes unavoidable that the process of front propagation takes place on a particular substrate whose structural details and/or microscopic irregularities cannot be properly described within a standard macroscopic formalism. On the other hand, it often happens that these structural features may represent key factors for the development of highly efficient materials. This is the case, for example, in the field of heterogeneous catalysis, where the morphological characteristics of the catalyst pore space can have a dramatic influence on the accessibility of the diffusion front of reagent towards the active sites in the deeper parts of the porous material [23]. In the extreme situation where the porous catalyst has a microscopically disordered, but macroscopically non-homogeneous geometry, even a departure from the classical diffusion formalism may be expected [24]. For example, the so-called anomalous type of 
transport in self-similar (fractal) structures usually occurs in the form of a subdiffusive regime $[25,26]$.

The invasion of magnetic flux into a disordered type II superconductor is another problem that has recently been the object of intense theoretical and experimental research. As a matter of fact, the magnetization properties of type II superconductors have been studied for many years, but the interest in this problem has been renewed with the discovery of high temperature superconductors $[27,28]$. The magnetization process is usually described in terms of the Bean model [29] and its generalizations: flux lines enter into the sample and, due to the presence of quenched disorder, give rise to a steady flux gradient. While the Bean model provides a consistent picture of average magnetization properties, such as the hysteresis loop and thermal relaxation effects [30], it does not account for local fluctuations in time and space. It has been recently observed that flux line dynamics is intermittent, taking place in avalanches [31], and flux fronts are not smooth [32-34]. In particular, it has been shown that the flux front crosses over from flat to fractal as a function of material parameters and applied field [33].

A widely used modeling strategy to describe the fluctuations around the Bean state consists of numerical simulations of interacting vortices, pinned by quenched random impurities [35-40]. With this approach it has been possible to reproduce flux profiles [37], hysteresis [37], avalanches $[36,39,40]$ and plastic flow $[35,40]$. One of the aims of these studies [37] is to establish precise connections between the microscopic models and the macroscopic behavior, captured for instance by generalized Bean models. A different approach treats the problem at mesoscopic scale, describing the evolution of interacting coarse-grained units [41, 42], supposed to represent the system at an intermediate scale. While these models give a faithful representation of several features of the problem, the connection with the underlying microscopic dynamics still represents a very active field of research.

\section{A. Invading front from an interacting particle simulation}

In this section, the main objective is to show that the gradient driven dynamics of the overdamped motion of interacting particles in disordered substrates can display a collective behavior that is typical of front invasion processes with roughened interfaces. More precisely, as we show next, the idea is to provide a basic description for these system in terms of a particle model and to indicate how the relevant scaling laws relating the front position and the flux profile to the pinning strength can be consistently extracted from numerical simulations. The equation of motion for an interacting particle performing an overdamped motion in a random pinning landscape can be written as in Eq. 1

$$
\Gamma \vec{v}_{i}=\sum_{j} \vec{J}\left(\vec{r}_{i}-\vec{r}_{j}\right)+\sum_{p} \vec{f}_{p}\left[\left(\vec{R}_{p}-\vec{r}_{i}\right) / \xi_{p}\right]+\eta\left(\vec{r}_{i}, t\right),
$$

where $\Gamma$ is the effective viscosity, the first term on the right hand side represents the particle-particle interaction, and the second accounts for the interaction between particles and pinning centers. Here we consider that $\vec{J}$ is a short-range interaction, and $\vec{f}_{p}$ is the force due to a pinning center, modeled as a localized trap at the position $\vec{R}_{p}$, with $\xi_{p}$ being the range of the wells (typically $\xi_{p} \ll \lambda$ ), and $p=1, \ldots, N_{p}$. For example, the pinning force could be modeled in terms of the expression, $\vec{G}(\vec{x})=-f_{0} \vec{x}(|\vec{x}|-1)^{2}$, for $|\vec{x}|<1$ and zero otherwise. For completeness, an uncorrelated thermal noise term $\eta$ with zero mean and variance $\left\langle\eta^{2}\right\rangle=k_{b} T \Gamma \delta\left(t-t^{\prime}\right)$ is also added to the equation, but we will restrict ourselves to the analysis of the case $T=0$ (see Ref. [43] for the implementation of thermal noise in simulations).

For gradient driven systems, the solution and interpretation of the MD model is essentially accomplished by the simultaneous numerical integration of Eq. (7) for each moving particle in the system, and subsequent analysis of the flux front propagation for different values of the pinning strength $f_{0}$. For instance, one typical simulation [46] can involve up to $N_{p}=800000$ Poisson distributed pinning centers of width $\xi_{p}=\lambda / 2$ in a system of size $\left(L_{x}=800 \lambda, L_{y}=\right.$ $100 \lambda)$, corresponding to a density of $n=10 / \lambda^{2}$. The number $N$ of flux lines depends essentially on the boundary condition adopted in the simulation. The injection of particles into the sample is implemented through the concentration at $t=0$ of all particles in a small slice $L^{\prime} \ll \lambda$, parallel to the $y$ direction, and imposing periodic boundary conditions in both directions. Due to mutual repulsion in the dense zone of the slice, the particles will be pushed inside the material, forming a penetrating front. The front position can then be taken as the $x$ coordinate of the most advanced particle in the system at different times, or one can divide the system into a grid and identify the front (see Fig. 1).

\section{B. Non-linear diffusion}

Now we show that the front penetration due to the collective motion of interacting particles in a substrate of pinning centers can be described by a disordered non-linear diffusion equation [51]. The equation can be obtained performing a coarse-graining of Eq. (7), starting from the FokkerPlanck equation for the probability distribution of the flux line coordinates $P\left(\vec{r}_{1}, \ldots, \vec{r}_{N}, t\right)$

$$
\Gamma \frac{\partial P}{\partial t}=\sum_{i} \vec{\nabla}_{i}\left(-\vec{f}_{i} P+k_{B} T \vec{\nabla}_{i} P\right),
$$

where $\vec{f}_{i}$ is the force on the particle $i$ given by Eq. (7). Next, we introduce the single particle density $\rho(\vec{r}, t) \equiv$ $\left\langle\sum_{i} \delta^{2}\left(\vec{r}-\vec{r}_{i}\right)\right\rangle$, where the average is done over the distribution $P\left(\vec{r}_{1}, \ldots, \vec{r}_{N}, t\right)$. The evolution of $\rho$ can be directly obtained from Eq. (8) and is given by 

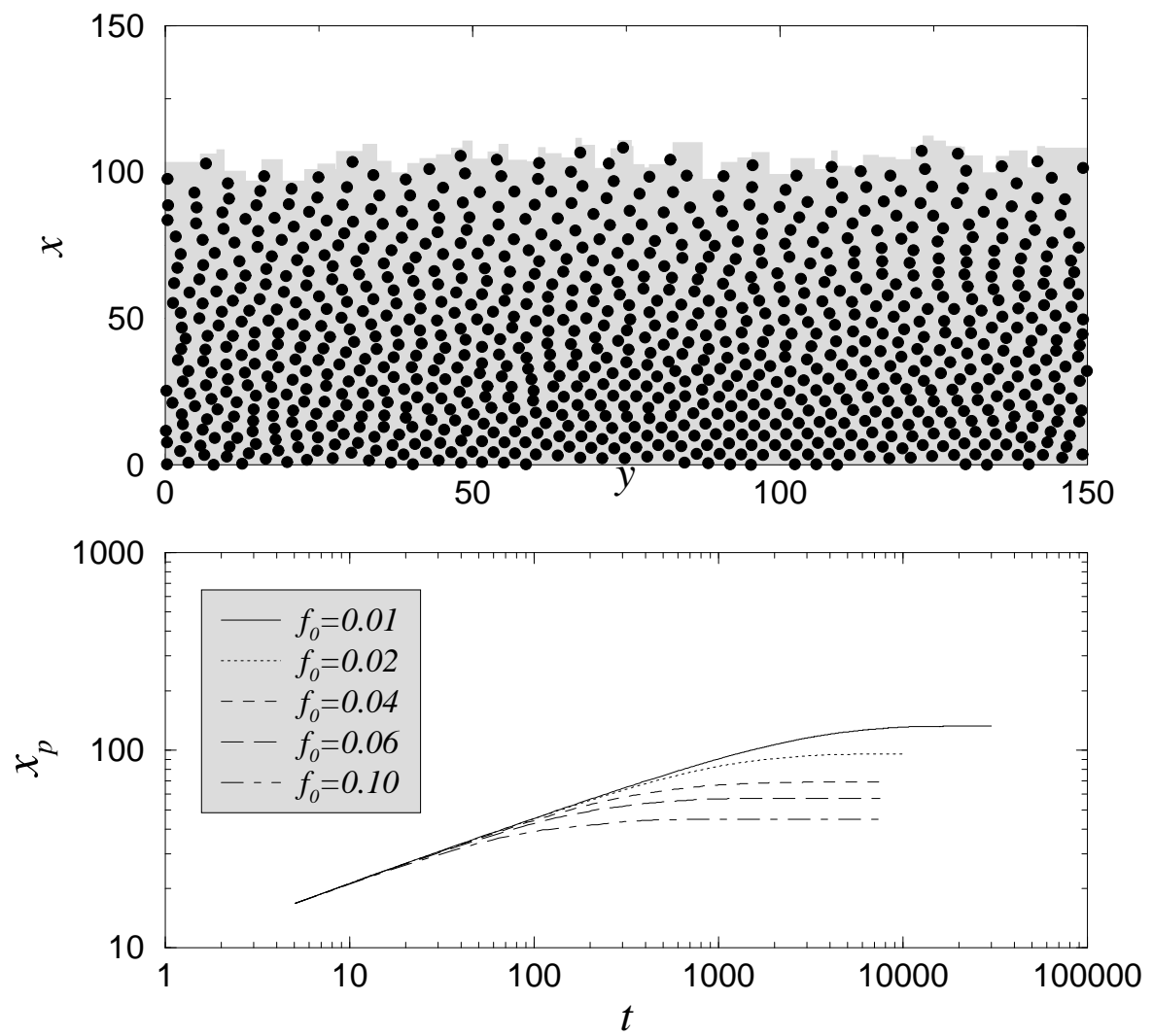

Figure 1. In the upper panel we show a typical realization of a flux front obtained from a simulation of interacting vortices in a disordered landscape [46]. In the lower panel, we show the growth of the average front position as a function of time for different pinning force strengths. The front initially grows as $t^{1 / 3}$ and then is pinned at a distance that scales with $f_{0}$.

$$
\Gamma \frac{\partial \rho}{\partial t}=-\vec{\nabla}\left(\int d^{2} r^{\prime} \vec{J}\left(\vec{r}-\vec{r}^{\prime}\right) \rho^{(2)}\left(\vec{r}, \vec{r}^{\prime}, t\right)+\sum_{p} \vec{f}_{p}\left[\left(\vec{R}_{p}-\vec{r}\right) / \xi_{p}\right] \rho(\vec{r}, t)\right)+k_{B} T \nabla^{2} \rho,
$$

where $\rho^{(2)}\left(\vec{r}, \vec{r}^{\prime}, t\right)$ is the two-point density, whose evolution depend on the three-point density and so on. The simplest truncation scheme involves the approximation $\rho^{(2)}\left(\vec{r}, \vec{r}^{\prime}, t\right) \simeq \rho(\vec{r}, t) \rho\left(\vec{r}^{\prime}, t\right)$. We then coarse grain the equation considering length scales larger than $\lambda$. This can be done expanding $\vec{J}$ in Fourier space, keeping only the lowest order term in $\vec{q}$, and retransforming back in real space. The result reads

$$
\int d^{2} r^{\prime} \vec{J}\left(\vec{r}-\vec{r}^{\prime}\right) \rho\left(\vec{r}^{\prime}, t\right) \simeq-a \vec{\nabla} \rho(\vec{r}, t)
$$

where $a \equiv \int d^{2} r \vec{r} \cdot \vec{J}(\vec{r}) / 2$.

The coarse graining of the disorder term is more subtle. A straightforward elimination of short wavelength modes would give rise, as in the previous case, to a random force $\vec{F}_{c}(\vec{r})=-g \vec{\nabla} n$, where $n$ is the coarse grained version of the microscopic density of pinning centers $\hat{n}(\vec{r}) \equiv \sum_{p} \delta^{2}(\vec{r}-$ $\vec{R}_{p}$ ) and $g \propto f_{0}$. This method can not be applied for shortrange attractive pinning forces as the one we are investigating. In this case, short wavelength modes yield a macroscopic contribution to pinning that can not be neglected.
Consider for instance the flow between two coarse grained regions: short-range microscopic pinning forces give rise to a macroscopic force that should always oppose the motion, while the random force derived above could in principle point in the direction of the flow. In other words, $F_{c}(\vec{r})$ should be considered as a friction force whose direction is always opposed to the driving force $\vec{F}_{d}$ (in our case $\left.\vec{F}_{d}=a \vec{\nabla} \rho\right)$ and whose absolute value is given by $|g \vec{\nabla} n|$ for $\left|\vec{F}_{d}\right|>|g \vec{\nabla} n|$ and to $\left|\vec{F}_{d}\right|$ otherwise [44].

Collecting all the terms, we finally obtain a disordered non-linear diffusion equation for the density of particles

$$
\Gamma \frac{\partial \rho}{\partial t}=\vec{\nabla}\left(a \rho \vec{\nabla} \rho-\rho \vec{F}_{c}\right)+k_{B} T \nabla^{2} \rho
$$

Any solution of Eq. (11) is clearly dependent on the particular boundary condition (BC) imposed to the system. It is therefore important to study the effect of different BC's on the dynamics of front propagation and to show that the macroscopic approach based on the coarse-grained expression Eq. (11) is compatible with the MD model. To be 
specific, we consider the situation in which the front penetration takes place in a disordered type II superconductor and the particles are vortices interacting according to Eq. (3) $[46,45]$. The following BC's correspond to different experimental situations $[47,48]$ :

- (A) Constant total number of vortices. Experimentally this corresponds to an external control of the magnetic flux.

- (B) Constant vortex concentration at the boundary. This case corresponds to an external control of the magnetic field.

- (C) Total vortex number increasing at constant rate. This represents an external control of the flux rate.

- (D) Boundary concentration increasing at constant rate, corresponding to a constant field rate.

One should notice that boundary conditions can be more complicated in reality, due to complex surface barriers that oppose flux penetration.

For a clean system $\left(f_{0}=0\right)$ at $T=0$, Eq. (11) can be solved exactly using scaling methods $[47,48]$. In this case, the density profiles obey the equation

$$
\rho(x, y, t)=t^{-\chi} \mathcal{G}\left(x / t^{\psi}\right),
$$

where $\chi$ and $\psi$ satisfy $\chi+2 \psi=1$. For the BC's considered: A) $\chi=1 / 3, \psi=1 / 3$; B) $\chi=0, \psi=1 / 2$; C) $\chi=-1 / 3, \psi=2 / 3$; and D) $\chi=-1, \psi=1$. These exponents are in perfect agreement with the results from MD simulations reported in Ref. [46]. For instance, the data reported in Fig. 1 correspond to boundary condition (A) and correctly scale with $\psi=1 / 3$. The function $\mathcal{G}(u)$ also depends on the boundary condition and for the case (A) is given by $\mathcal{G}(u)=\left(1-u^{2}\right) / 6$ for $u<1$ and vanishes for $u \geq 1$. The other cases are reported in Refs. [47, 48].

The presence of disorder (pinning centers) induces substantial effects on the behavior of the system that can be quantified in terms of the front propagation and/or the shape of the density profiles of flux lines. Depending on the boundary conditions, it has been observed that the front is either pinned or simply slowed down [45]. Extensive numerical simulations have also been performed in Refs. [45, 46] to show the compatibility between the MD model with disorder and its coarse-grained representation, Eq. (11). Moreover, by varying the parameters of this continuum description of the front propagation, a crossover from flat to fractal flux fronts has been detected, consistent with experimental observations. The value of the fractal dimension suggests that the strong disorder limit is described by percolation. In the weak disorder limit, we recover the analytical results derived in Refs. [47, 48].

\section{Externally driven dynamics: de- pinning and flow}

In this section, we discuss the response of interacting particles to an external force in presence of quenched disorder.
The effect of quenched disorder is first analyzed in a single particle model, which, although oversimplified, still displays a depinning transition. The effect of the interparticle potential on the depinning transition will be first introduced in the framework of the elastic theory, which breaks down for strong pinning, leading to plastic depinning. Finally, we briefly discuss the moving phases observed for strong driving.

\section{A. Single particle pinning}

A first understanding of the dynamics of driven particles in a disordered landscape can be obtained considering the motion of a single particle [49-51]. We consider a collection of parabolic wells for the pinning potentials, so that the equation of motion for a single particle is given by

$$
\Gamma \frac{d x}{d t}=F+\sum_{p} f_{0}\left(X_{p}-x\right) \theta\left(\left|x-X_{p}\right|-\xi_{p}\right),
$$

where are the random coordinates of the pinning centers, which we assume to be non-overlapping. When the particle is not interacting with a pinning center, it moves with constant velocity $F / \Gamma$, until it enters into the attraction range of a pinning center. Considering this as the initial condition (i.e. $x(t=0)=X_{p}-\xi_{p}$ ), we can solve the equation

$$
\Gamma \frac{d x}{d t}=F+f_{0}\left(X_{p}-x\right) \quad x<X_{p}+\xi_{p},
$$

which is given by

$$
x(t)=X_{p}+F / f_{0}-\left(F / f_{0}+\xi_{p}\right) e^{-t / \Gamma} .
$$

In the limit $t \rightarrow \infty$ the particle remains trapped as long as $F<F_{c}=\xi_{p} f_{0}$ and escapes otherwise. We can interpret this behavior as a depinning transition: the particle is pinned for $F<F_{c}$ and moves for $F>F_{c}$.

The force-velocity diagram can be computed noting that for $F>\xi_{p} f_{0}$ the particle spends in each trap a time

$$
\tau=\Gamma \log \left(\left(F+F_{c}\right) /\left(F-F_{c}\right)\right) .
$$

The total time $T$ to cross a system of length $L$ with $N_{p}$ pinning centers is given by

$$
T=N_{p} \tau+\left(L-2 \xi_{p} N_{p}\right) \Gamma / F .
$$

The average velocity of the particle is thus given by

$$
v=\frac{L}{T}=\frac{F}{\Gamma\left(F n_{p} \log \left(\left(F+F_{c}\right) /\left(F-F_{c}\right)\right)+\left(1-2 \xi_{p} n_{p}\right)\right)},
$$

where $n_{p}=N_{p} / L$ is the number of pinning centers per unit length. Close to the depinning transition $F_{c}$ the velocity scales $\operatorname{logarithmically~} v \sim-1 / \log \left(F-F_{c}\right)$, while at higher forces the velocity is proportional to the force. This behavior is characteristic of the depinning transition, but in general $v \sim\left(F-F_{c}\right)^{\beta}$, where $\beta$ is a non trivial critical exponent. Notice that the logarithmic behavior found above is an artifact of the discontinuous pinning force. Using a continuous force, one obtains instead a behavior of the type 
$v \sim\left(F-F_{c}\right)^{1 / 2}$ [50]. We notice that the dependence of the exponents on the pinning potential is a peculiarity of the single particle behavior and is not observed when interactions come into play as we will discuss in the next section.

Single particle models are also useful for gradient driven dynamics discussed in the previous section [46]. In that case the external force $F$ is replaced by the density gradient, which can be approximated with a simple function of $x$. For instance, in the case of boundary condition (B) (see section III) $F \sim \nabla \rho \sim \rho_{0} / x$, where $\rho_{0}$ is the boundary density. This simple model yields the correct scaling behavior for the front position [46].

\section{B. Elastic depinning}

In the previous section, we have only considered the interactions between moving particles and static pinning centers. We could expect that those results will be valid only when the interactions between particles can be neglected, as for instance in a very dilute system. In most situations, however, interactions between particles should be explicitly taken into account. In this case we will still observe a depinning transition, but its quantitative and qualitative features will change [52-58]. It is convenient to first study an interacting particle system in the elastic approximation, in which the pinning forces are not strong enough to break the topological properties of the particle system. For instance, if the particles are arranged into a crystal, the external force and the disorder preserve the topological order and no defects are generated.

In the pinned phase we can write the positions of the particles by their displacement vectors $\vec{u}\left(\vec{R}_{i}\right)=\vec{r}_{i}-\vec{R}_{i}$, where $\vec{r}_{i}$ are the coordinates in the deformed system and $\vec{R}_{i}$ are the equilibrium positions. The particle interaction energy can then be expanded in terms of the displacement field, which is assumed to be small,

$$
U=\sum_{i j} V\left(\vec{r}_{i}-\vec{r}_{j}\right) \simeq U_{0}+\sum_{\alpha, \beta} \sum_{i j}\left(u_{\alpha}\left(\vec{R}_{i}\right)-u_{\alpha}\left(\vec{R}_{j}\right)\right) \frac{\partial^{2} V}{\partial r_{\alpha} r_{\beta}}\left(u_{\beta}\left(\vec{R}_{i}\right)-u_{\beta}\left(\vec{R}_{j}\right)\right)
$$

where $U_{0}$ is the energy in equilibrium. One can then take the continuum limit expanding in small gradient $u(\vec{R}) \simeq$ $u\left(\vec{R}^{\prime}\right)+\left(\vec{R}-\vec{R}^{\prime}\right) \cdot \vec{\nabla} u(\vec{r})$ and obtain [59]

$$
U=U_{0}+\frac{1}{2} \int d^{3} r \sum_{\alpha, \beta \gamma \delta} E_{\alpha, \beta \gamma \delta} \frac{\partial u_{\alpha}}{\partial r_{\gamma}} \frac{\partial u_{\beta}}{\partial r_{\delta}},
$$

where the elastic tensor $E_{\alpha, \beta \gamma \delta}$ can be expressed in terms of the interparticle pair potential as

$$
E_{\alpha, \beta \gamma \delta}=-\frac{1}{2} \sum_{R} R_{\gamma} R_{\delta} \frac{\partial^{2} V}{\partial r_{\alpha} r_{\beta}}
$$

Clearly this expansion holds as long as the sum in Eq. 21 converges. If interactions are long range, decaying with a slow power law $V(r) \sim r^{-(d-1+\sigma)}$ with $\sigma<2$, the elastic energy can not be expanded in gradients and we have to live with a non-local interaction.

Coming back to the local limit, a further simplification is obtained in Eq. (20) if we take into account the symmetries of the equilibrium system. In the case of an isotropic system, the elastic tensor has only two independent components and the energy reduces to

$$
U=U_{0}+\frac{1}{2} \int d^{3} r K\left(\frac{\partial u_{\alpha}}{\partial r_{\alpha}}\right)^{2}+\mu\left(\frac{\partial u_{\alpha}}{\partial r_{\beta}}\right)^{2},
$$

where $K$ and $\mu$ are the compression and shear moduli, respectively.

Using the elastic expression for the interparticle energy, we can rewrite the equation of motion for the particles as

$$
\frac{\partial u_{\alpha}}{\partial t}=\mu \nabla^{2} u_{\alpha}+(K+\mu) \frac{\partial}{\partial r_{\alpha}}(\vec{\nabla} \cdot \vec{u})+F+f_{\alpha}(r, u) .
$$

When expanding around a moving state, we obtain the same equation with an extra convective term $\vec{v} \cdot \vec{\nabla} u$ on the left hand side $[60,61]$. These elastic equations are still impossible to solve exactly, but several results have been obtained using scaling theories and renormalization group calculations [5258]. In the case of long-range interactions the gradients are replaced by a non-local interaction kernel and the equation becomes

$\frac{\partial u_{\alpha}}{\partial t}=\int d^{d} r^{\prime} K_{\alpha \beta}\left(r-r^{\prime}\right)\left(u_{\beta}\left(r^{\prime}\right)-u_{\beta}(r)\right)+F+f_{\alpha}(r, u)$,

with $K_{\alpha \beta}(r) \sim 1 / r^{d+\sigma}$ for large distances.

A first insight on the behavior of interacting particles in a disordered media in the elastic approximation can be gained by collective pinning theory, originally due to Larkin [62]. In this approach, the main energetic contributions entering the problem are written as a function of a lengthscale $L$. Minimizing the sum of elastic and disorder energies, one obtains the characteristic length $L_{c}$ at which pinning becomes relevant. Considering a region of size $L$, where the typical displacements of the elastic medium are of the order of the range $\xi_{p}$ of the pinning potential, the elastic energy can be estimated as $E_{e l} \sim \mu L^{d-2} \xi_{p}^{2}$, where for simplicity we have only considered the shear modulus $\mu$. The pinning energy can be estimated in the limit of weak pinning, when the main effect comes from the fluctuations in the disorder: in a region of size $L$, the typical fluctuations of the pinning energy scale as $E_{\text {pin }} \sim-E_{p} \sqrt{L^{d} n_{p}}$, where $E_{p}=f_{0} \xi_{p}, n_{p}$ is the density of pins. Minimizing the total 
energy $\mu L^{d-2} \xi_{p}^{2}-E_{p} \sqrt{L^{d} n_{p}}$ with respect to $L$, we obtain the Larkin length

$$
L_{c} \sim\left(\frac{\mu \xi_{p}^{2}}{\sqrt{n_{p}} E_{p}}\right)^{\frac{2}{4-d}} .
$$

For $L<L_{c}$ the elastic medium is essentially undeformed, while elastic deformations grow strongly for $L>L_{c}$ and typically, the system becomes rough on large lengthscales.

Notice that the Larkin length decreases with the disorder strength as long as $d<d_{c}=4$. The dimension $d_{c}=4$ enters here for the first time, but has in fact a great importance. As can be shown by renormalization group calculations, $d_{c}$ is the upper critical dimension: for $d>d_{c}$ the system does not roughen and the critical behavior at the depinning transition is essentially mean-field like. Since the physical dimension is usually lower than four, one is lead to think that this limit is without practical interest. This is not completely true, since in presence of long-range interactions $d_{c}$ typically lowers, reaching sometimes the physical dimension. In particular, for non local elastic interactions with $\sigma<2$ the upper critical dimension is given by $d_{c}=2 \sigma$.

Since $L_{c}$ represents the characteristic lengthscale at which pinning starts to be effective, Larkin estimated the depinning threshold as the force needed to unpin a region of length $L_{c}$. This can be done comparing the energy due to the external force $E_{\text {ext }}=F \xi_{p} L^{d}$ to the pinning energy at the scale $L_{c}$, obtaining

$$
F_{c} \sim E_{p} \sqrt{n_{p}} L_{c}^{-d / 2} / \xi_{p}=\frac{\left(f_{p \sqrt{n_{p}}}\right)^{4 /(4-d)}}{\left(\xi_{p} \mu\right)^{d /(4-d)}} .
$$

Collective pinning theory provides a qualitative description of the depinning transition and a good estimate of the depinning threshold, but does not allow to estimate the critical exponents and the force-velocity curve. For this, one needs renormalization group methods, which we do not want to discuss here. We just quote some results, obtained for the first order in a $\epsilon=4-d$ expansion: the exponent for the velocity scaling is given by $\beta=1-\epsilon / 9$, while the roughness exponent, ruling the scaling of the typical displacements with the system size $u \sim L^{\zeta}$, is estimated as $\zeta=\epsilon / 3$. Other exponents follow from scaling relations. For instance, one can analyze the transient behavior of the average particle velocity, which initially decays as a power law $\langle v\rangle \sim t^{-\alpha}$, and then crosses over to a constant value when $F>F_{c}$, or goes exponentially to zero otherwise (see Fig. 2) [51]. The exponent $\alpha$ can be obtained by a scaling relation as $\alpha=\beta / \nu z$, where $z$ is the dynamic exponent and $\nu$ is the correlation length exponent [51].

Simulations have been widely used in the past to obtain numerically the value of the exponents. We should distinguish here the vast body of work pertaining to elastic manifolds, in which the elastic approximation is enforced directly in the model simulating variants of Eq. 23 [55], from particle simulations in which the original system is studied. In one dimension the elastic approximation works well and the exponents measured in particle models reproduce with a good accuracy the results obtained for elastic manifolds, namely $\beta \simeq 0.25$ and $\zeta \simeq 1.25$ (see Fig. 3) [51,63-65]. This is not surprising since in $d=1$ topological order is trivially present. In two dimensions from the mapping with elastic manifold we would expect $\beta \simeq 0.65$ and $\zeta \simeq 0.75$ [55] and at least the first exponent is reproduced by particle simulations [66].

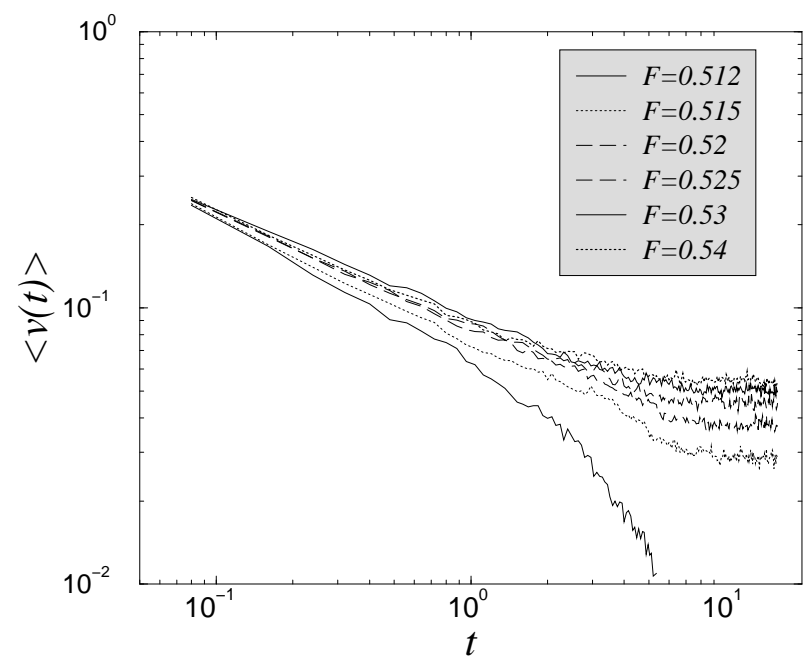

Figure 2. The decay of the velocity for $N=400$ interacting particles with pinning in $d=1$ for different values of the force. For $F>F_{c}=0.514$ the velocity crosses over to a steady value. See Ref.[51] for details on the model.

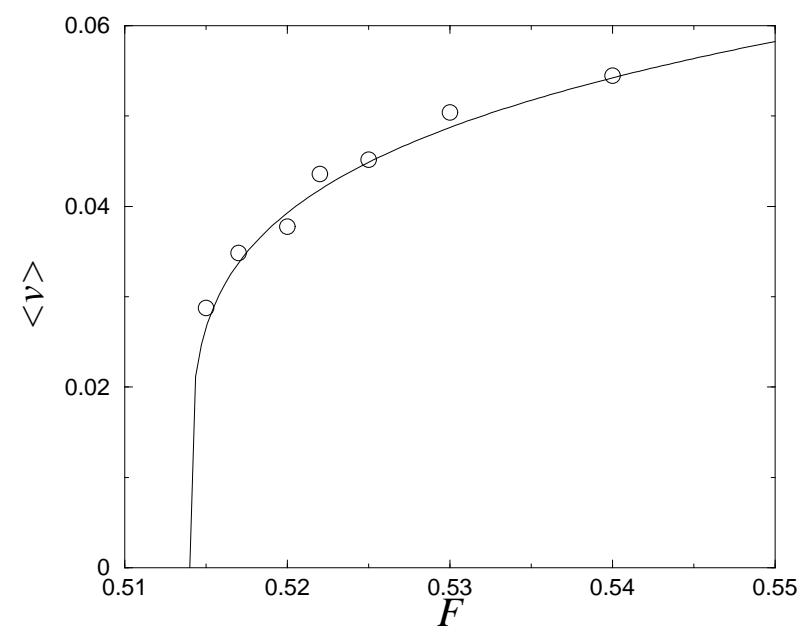

Figure 3. The force velocity curve obtained from the steady state velocity shown in Fig. 2. with pinning in $d=1$. The best fit yields $\beta=0.22$ and $F_{c}=0.514$ [51].

\section{Plastic depinning}

When pinning forces become stronger and/or pinning centers more dilute the topological order of the system typically breaks down. In this case, it is not possible to describe the deformation in terms of a displacement field as we did in the previous section, and plastic deformation should be explicitly considered. Due to these difficulties, a complete theoretical understanding of plastic depinning is still not available and one should rely on numerical simulations. A simple estimate of the conditions for the occurrence of strong pinning effects can be gained in the framework of collective pinning theory. When $L_{c} \sim a_{0}$, where $a_{0}$ is the interparticle distance, the particles are pinned individually. One can 

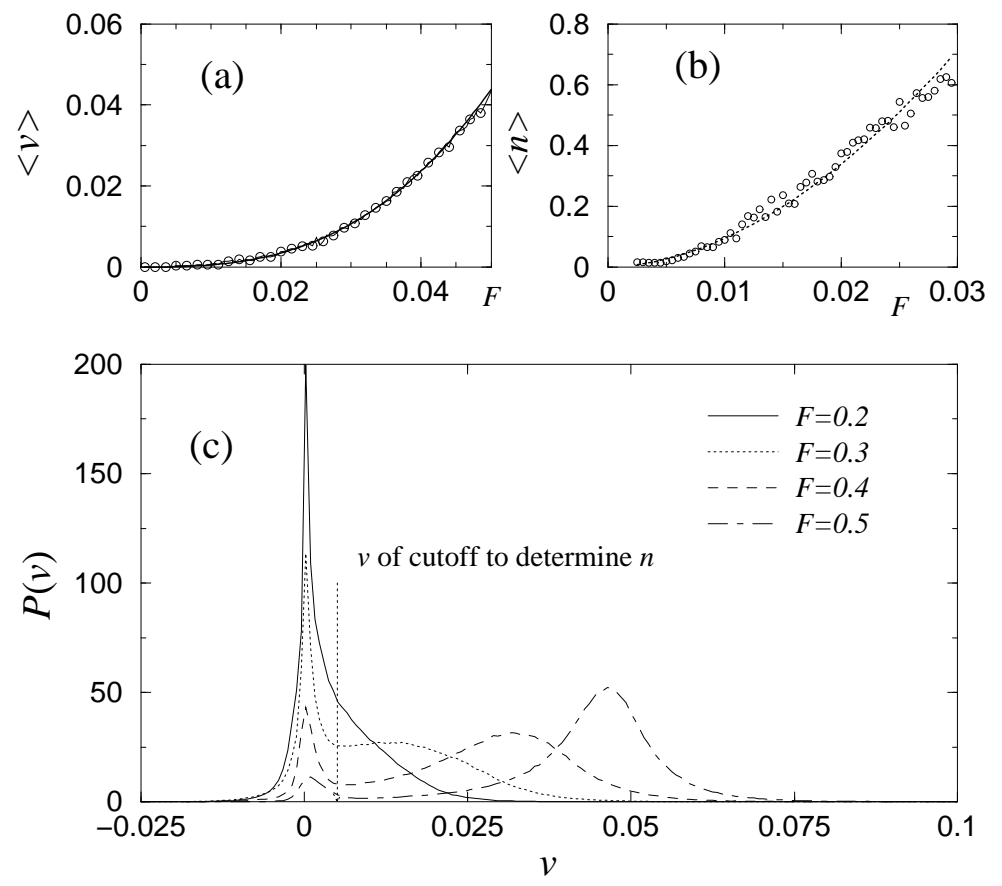

Figure 4. (a) The force velocity curve for $N=270$ interacting particles in the strong pinning limit in $d=2$. The fit is a power law $F^{\beta}$ with $\beta=2.7$. (b) The velocity distribution for the same system for different values of the force. Notice the bimodal structure that can be used to set a threshold and identify moving particles. (c) The average number of moving particles scaling with the applied force as $F^{\gamma}$ with $\gamma=1.8$.

elaborate these type of arguments and draw phase diagrams in terms of disorder and applied force [67].

Here we discuss the main features of plastic depinning as observed in numerical simulations. Typically the force velocity curve differs drastically from the one observed when depinning is elastic, which is upward convex (see Fig. 3). In plastic depinning, the curve is convex downward implying that $\beta>1$. For instance Ref. [66] finds $\beta=2.2$ in a simulation of colloids. Others do not provide a value for $\beta$ since it appears that a precise estimate of the exponent is not straightforward [67]. The main reason is that the depinning force $F_{c}$ is small and one could as well believe that $F_{c}=0$ for a large system, and that the pinning seen in simulations could be an artifact of finite sizes. For instance, the curve reported in Fig. 4 can be reasonably fit as $v \sim F^{\beta}$, with $\beta \simeq 2.7$. A small value of $F_{c}$ would not change significantly this fit but it is difficult to discriminate between the two cases.

The important differences between plastic and elastic depinning can be highlighted computing the velocity distribution of the moving particles. In plastic depinning the solid breaks apart: some particles are pinned by the strong defects while others move, typically in channels. Thus the velocity distribution develops two peaks: one around zero corresponding to pinned particles, and one at higher velocities [67]. Using this bimodal distribution, it is possible to identify the fraction of moving particles $\left\langle n_{m}\right\rangle$, using a velocity threshold situated in the middle of the two peaks (see Fig. 4). Again the data are reasonably fit by a power law $F^{\gamma}$ with $\gamma \simeq 1.8$.

The channel structure of the dynamics has been studied thoroughly in the literature analyzing the statistical proper- ties of particle trajectories [35, 40, 71]. In Fig. 5 we report an example of the trajectories in the plastic regime, showing the separation between pinned and moving particles. It is also possible to analyze the tearing of the lattice studying its topological properties (i.e. the presence of defects, such as dislocations) [67, 71, 72].

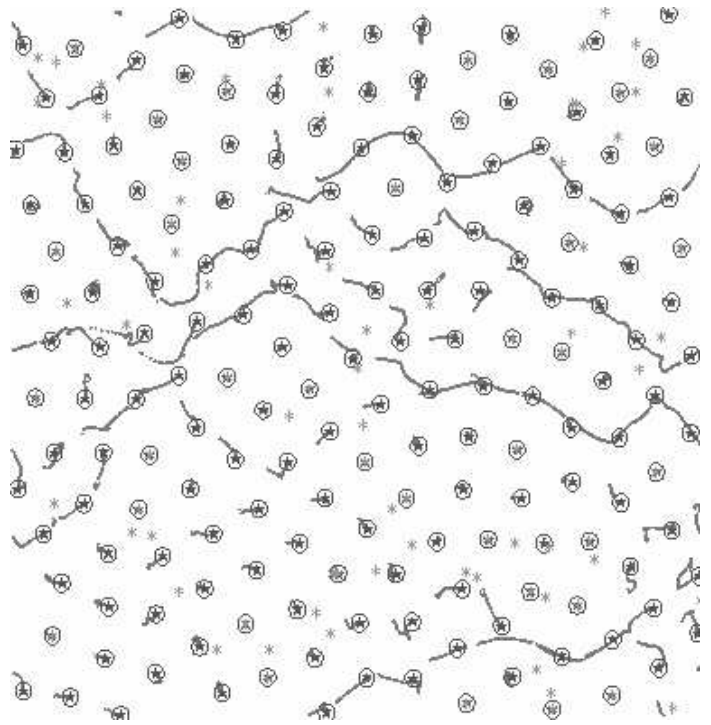

Figure 5. An example of particle trajectories in the plastic regime. Particles are depicted by circles, pinning centers by stars and trajectories by lines.

To understand theoretically plastic depinning several authors have introduced simplified lattice models, which are possibly amenable to analytical treatment. For instance, Refs. [73, 74] propose a model in which particles flow in 
a two dimensional rotated square lattice. When the number of particles present in a site is larger than a threshold, one particle is transferred to a neighboring site. The flow is directed and the outlet of each site is random but fixed. It is interesting to note that this model is equivalent to a sandpile model [76, 77], namely a random (quenched) directed Manna model [78, 79]. It should be possible to use the result obtained for the directed Manna model to solve exactly this model [80, 81]. The model displays a plastic depinning transition: some particles are trapped and others flow in channels. The number of particles belonging to the flow basin scales as $n_{m} \sim\left(F-F_{c}\right)^{\gamma}$, with $\gamma \simeq 0.5$. The average particle current (or velocity) scales instead as $v \sim\left(F-F_{c}\right)^{\beta}$ with $\beta=1.5$. It was conjectured that $\beta=1+\gamma$, implying that the velocity exponent is due to the combination of the scaling of the velocity of the flowing particle $v_{f}$ and the one of the channel size.

A similar reasoning was proposed in Ref. [75] for plastic depinning in a disordered XY model. In this model each spin depins as a single particle in a smooth pinning potential as $v_{s} \sim\left(F-F_{c}\right)^{1 / 2}$. The number of spins depinning also scale with the applied force as $n_{m} \sim\left(F-F_{c}\right)^{\gamma}$, yielding $\beta=\gamma+1 / 2$. The exponent $\gamma$ was found to be close to $d$, the dimension of the lattice, which suggests that a simple geometrical description could be possible. We notice that the results presented in Fig. 4 satisfy approximately the relation $\beta=1+\gamma$, although $F_{c} \simeq 0$.

Recently, a different approach for plastic depinning was discussed in Refs. [68, 69] through a model of a viscoelastic medium. In this model, depending on the parameter values, one observes first order type or continuous depinning. The model can be approached by mean-field theory [68] and renormalization group [69], but at present the connections with plastic depinning in particle systems is not clear. It is interesting to notice that a first order depinning transition, with hysteresis, is also expected in presence of inertia [70]. Raising the force from the pinned phase towards the moving phase is different than decreasing it when the system is already moving. In the latter case inertia will keep the system in motion even beyond the "depinning threshold", resulting in an hysteretic force velocity curve.

To summarize, plastic depinning is characterized in general by the tearing of the elastic medium through the production of dislocations. Only a fraction of the particles move along channels, while the others are pinned, suggesting to interpret the force velocity curve by a combination of scaling of single particle velocities and channel size. However, the wide fluctuations of the numerical values for the exponents present in the literature and the lack of a theory does not allow a clear quantitative picture of the phenomenon.

\section{Moving phases, hysteresis and avalanches}

When the external driving force is large enough, particles enter into a moving phase which can be of several kinds depending on the type of disorder and the structure of the particle system. All these aspects are reviewed in Ref. [82]. A first understanding of the dynamics of an elastic system for strong driving forces comes from a high velocity expansion [83]. When the system flows rapidly one can write $u(r, t) \simeq V t+\delta u$, where $V$ is the average flow velocity.
In a first approximation the pinning force becomes effectively a thermal like noise $f_{p}(r, u) \simeq f_{p}(t, V t)$, with an effective dynamic temperature decreasing with velocity as $T_{d} \sim 1 / V$. Thus one would expect that beyond a certain velocity the elastic system would reorder since the effect of pinning forces disappear. The discreteness and periodicity of a particle system modifies considerably this picture: while the displacements along the direction of motion do not feel the disorder due to the high velocity, this is not the case for transverse displacements. Taking into account this effect, Giamarchi and Le Doussal [60] show that the system should decouple in elastic channels and predict the existence of threshold for transverse depinning. These features have been then observed in simulations [72]. For an extensive discussion of other aspects of the dynamics we refer to Ref. [82]

So far we have only discussed the dynamics of interacting particles in random media occurring under a constant applied force. When the applied force is time dependent we observe other interesting phenomena. In particular, an AC drive leads to hysteresis, which has been studied in detail for domain walls in ferromagnetic materials [85, 86], it should be possible to carry over these results to a generic elastic system with disorder [87]. Hysteresis is expected in the quasistatic limit already at the level of a single particle model driven by an elastic spring [44]. Close to the depinning transition, the hysteresis loop of the average displacement as a function of the force can be obtained analytically solving the equation [85]

$$
\Gamma\langle d u / d t\rangle=\left(F_{0} \sin (\omega t)-F_{c}\right)^{\beta} \theta\left(F-F_{c}\right) .
$$

Finally, Ref. [88] shows that the velocity force curve also displays hysteresis, but only in the dynamic regime (i.e. hysteresis is lost in the limit $\omega \rightarrow 0$ when one recovers the usual force velocity depinning curve).

It is now well established that when the force is raised slowly towards the depinning threshold the dynamics of an elastic system takes place in the form of avalanches as in self-organized criticality [76]. In particular, the avalanche size distribution scales as

$$
P(s)=s^{-\tau} f\left(s / s^{*}\right),
$$

where the cutoff size grows as a power law with the distance from the critical point $s^{*} \sim\left(F_{c}-F\right)^{-\nu D}$. Here $\nu$ is the correlation length exponent and $D$ is the fractal dimension of the avalanche (i.e. $s^{*} \sim \xi^{D}$ ) where $\xi$ is the correlation length. The connection with self-organized criticality is more apparent when the elastic system is driven at "constant velocity" [65]. This can be achieved coupling the system elastically to a slider moving at constant velocity, or, in other words, replacing $F$ by $k\left(V t-\int d^{d} r d t \dot{u}(r, t)\right)$. In the limit of $V \rightarrow 0$ and $k \rightarrow 0$ the system is driven precisely at the depinning transition $[65,77]$. For a general review on avalanches and self-organized criticality see Ref. [77]. 


\section{Kinetically constrained dynamics: jamming and shear yielding}

In this section, we will discuss the flow behavior of a wide class of physical systems whose dynamics is governed by the presence of kinematical constraints induced by both interactions and geometry. One of the main features shared by their dynamics is the presence of jamming, a new concept recently proposed to refer to the suppression of the temporal relaxation of a physical system and its corresponding ability to explore the space of configurations $[89,90]$. Under the action of externally applied shear stresses, these systems eventually yield and are able to flow like a viscous fluid. Shear yielding is thus another feature they have in common.

As we will see in the following subsections, under stress conditions both soft materials [7] and crystalline solids [91] are susceptible to display jamming and shear yielding, due to the interactions and spatial arrangement of their constituent particles in the case of soft-matter systems, or to the interactions and spatial arrangement of their topological defects — such as dislocations - in plastically deforming crystals. Jamming and yielding could in turn be responsible for the remarkably similar creep and stationary flow rheology observed experimentally in these systems, in spite of the big differences among the materials involved.

\section{A. Jamming and viscoelastic flow in soft condensed mat- ter materials}

The phenomena of jamming and yielding control the behavior and properties of soft materials as diverse as colloidal suspensions [92], emulsions [93], foams [94], gels [95, 96], pastes [97], biological tissues [98], and other soft matter systems. Most of these physical systems consist of various types of soft particles closely packed into an amorphous state. At such high concentrations, the individual motion of particles is drastically constrained and, as a consequence, soft and concentrated materials usually respond like elastic solids upon the application of low stresses. On the other hand, they flow like viscous fluids above the so-called yield stress value $\sigma_{y}$, exhibiting a common rheology. The deformation process of amorphous polymeric materials has, for instance, received a great deal of attention [7]. The creep compliance curve of amorphous polymer networks above the glass transition temperature has been reported to closely exhibit the following behavior [99, 100]

$$
J(t)=\gamma / \sigma=j_{0}+C t^{1 / 3}+t / \eta,
$$

where $\gamma$ is the global strain of the material, $\sigma$ is the external stress, $j_{0}$ is the instantaneous elastic component of the compliance, and $\eta$ is the viscosity. According to this behavior, the $C t^{1 / 3}$ term, also known as Andrade creep term, dominates for times much smaller than the relaxation time $t_{c}$ characteristic of the complex fluid, while a macroscopic viscous flow of the form $t / \eta$ is established after much longer times $t>>t_{c}$. One can define and measure a time dependent effective viscosity in the Andrade creep regime $\eta=\sigma / \dot{\gamma} \sim t^{2 / 3}$, which reaches its equilibrium value at longer times.
A common nonlinear rheology is also observed for higher stress values, or shear strain-rate values in the case of constant strain-rate experiments which are in most cases performed in this class of systems. The stress-strain relationships in the steady state are often described by phenomenological equations of the form $\sigma=\sigma_{y}+a \dot{\gamma}^{n}$ [101, 102], which imply a nonlinear dependence of the stress $\sigma$ on the shear strain rate $\dot{\gamma}$. For a Newtonian dispersion $\sigma_{y}=0$ and $n=1$, resulting in a constant viscosity coefficient $\eta=\sigma / \dot{\gamma}$. If instead $\sigma_{y} \neq 0$, the equation describes a Bingham fluid. When $n<1$ the relation is known as the Hershel-Bulkeley law, but if $\sigma_{y}=0$, the equation describes a power-law fluid, with a shear strain rate decreasing viscosity.

Although the understanding of yield and viscoplastic flow in these materials is difficulted by the absence of a clear mediating mechanism, such as the motion of dislocations in a crystal, it has been argued that the common rheology displayed by these general class of complex fluids might be attributed to two particular shared properties of these materials: structural disorder and metastability; which are characteristic features of an underlying glassy dynamics [103]. In a few words, a glassy dynamics is associated to the slow structural relaxation which takes place when some parts of the system are trapped by their neighbors and have to surmount large energy barriers to explore further more favorable configurations. Molecular dynamics simulations [104, 105, 106, 107] of glass-forming liquids and polymers have proved of much help in this respect. About the same time, a general "jamming" scenario was also proposed [89] as a common framework to understand the mechanical behavior of a broader class of nonequilibrium physical systems (colloidal suspensions, supercooled liquids, foams, etc. and granular media) which, in spite of their differences, exhibit common properties such as slow dynamics and scaling features near the so-called jamming threshold.

Regarding the slow relaxation dynamics characteristic of soft glassy materials, recent experiments [108, 109] show the necessity of incorporating dynamical heterogeneities for its complete description. In this respect, a new light scattering method, introduced in Ref. [96], allowed to detect the intermittent dynamics of a gel formed from attractive colloids. The dynamics is found to be intermittent due to random rearrangements which appear to be localized in time. This and similar experiments strongly suggest that intermittent behavior seems to be a fundamental ingredient for the slow relaxation in jammed materials.

Finally, it is interesting to point out that an empirical relation known as the "Cox-Merz rule" [110] quite successfully relates the non-linear steady rheology with the linear but frequency dependent rheological properties of polymer melts. In particular, the Cox-Merz rule relates the steady viscosity at a given shear rate $\dot{\gamma}$, to the modulus of the dynamic viscosity at a frequency $\omega=\dot{\gamma}$, i.e. $\eta(\dot{\gamma})=\left|\eta^{*}(\omega)\right|$. Several works have a posteriori tried to theoretically justify this empirical relation starting from basic assumptions [111]. Indeed, the time dependent rheology which follows from Eq. (29) in the Andrade regime $\eta=\sigma / \dot{\gamma} \sim t^{2 / 3}$, is dimensionally equivalent to the shear-thinning behavior $\eta \sim$ 
$\dot{\gamma}^{-2 / 3}$ reported in Ref. [106] for the steady nonlinear rheology of a binary Lennard-Jones mixture, and it would be consistent with the Cox-Merz rule for $w=1 / t$. The shear thinning exponent could also be related to the subaging behavior observed in many soft-glassy materials $\eta \sim t_{w}^{\mu}$, where $t_{w}$ is the so-called waiting time and $\mu<1$ [112].

In the following section, we will show that most of the attributes discussed for the case of amorphous soft-glassy materials are also shared by crystalline materials like soft metals or vortex lattices deforming plastically due to the motion of dislocations.

\section{B. Dislocation jamming and viscoplastic creep deforma- tion}

The viscoplastic deformation of crystalline solids is due to the creeping motion of dislocations driven by an externally applied stress $[113,114,12,115]$. The study of the dynamics of these linear topological defects is a subject of considerable interest because of its practical importance in materials design and engineering. It is also interesting from the theoretical point of view for the many features that dislocation motion shares with other complex systems like, for instance, flux lines in high temperature superconductors, or some of the soft matter materials discussed above.

At the beginning of the XX century, Andrade reported that the creep deformation of soft metals at constant temperature and stress grows in time according to a power law with exponent $1 / 3$, i.e. $\gamma \sim t^{1 / 3}$ where $\gamma$ is the global strain of the material [116]. More generally, the creep deformation curve usually follows the relation $\gamma(t)=\gamma_{0}+\beta t^{1 / 3}+\kappa t$, where $\gamma_{0}$ is the instantaneous plastic strain, $\beta t^{1 / 3}$ is known as Andrade creep, and $\kappa t$ is referred to as linear creep regime $[113,114]$. The same qualitative behavior has since been observed in many materials with rather different structures leading to the conclusion that this should be a process determined by quite general principles, independent of most material specific properties. Notice that the creep curve for amorphous polymer melts introduced in the previous subsection, follows exactly the same relation. Various arguments have been proposed within the dislocation literature [113,114,117-119] to try to explain Andrade's creep. Most of them are based on thermally activated processes over time (or strain) dependent barriers, however, there is still a lack of consensus on the basic mechanism involved in the phenomenon.

As in the case of soft-matter systems, the plastic deformation of crystals only occurs when the externally applied stress overcomes a threshold value, the yield stress of the material. Above this threshold value, large-scale dislocation motion may take place, and a steady regime of plastic deformation is eventually established. Dislocations tend to move cooperatively under the action of external stress due to their mutual long-range and anisotropic elastic interactions, which can be attractive or repulsive. As a result of these interactions, and of the spatial dislocation structures they give rise to, self-induced constraints build up in the system and the motion of dislocations may eventually cease. Nevertheless, small variations of the external loading, the density, the dislocation distribution or the temperature can enhance dislocation motion in a discontinuous and intermittent manner [120].

In Ref. [91], the temporal relaxation of a relatively simple dislocation dynamics model was studied through numerical simulation. In particular, a collection of parallel straight edge dislocations with Burgers vectors $\mathbf{b}_{i}=b \hat{x}$ moving in a single slip system under the action of constant stress was shown to give rise to Andrade-like creep at short and intermediate times for a wide range of applied stresses, without invoking thermally activated processes, i.e. $T=0$ (see Fig. 6). The strain rate, which is proportional to the density of mobile dislocations $d \gamma / d t=\sum_{i} b_{i} v_{i}$ with $v_{i}$ the velocity of each dislocation, decays as a power law with an exponent close to $2 / 3$, in agreement with Andrade's observations. At larger times, the strain-rate was observed to cross over to a linear creep regime (i.e. to a plateau signaling a steady rate of deformation) whenever the applied stress is larger than a critical threshold $\sigma_{c}$, or, otherwise, to decay exponentially to zero.

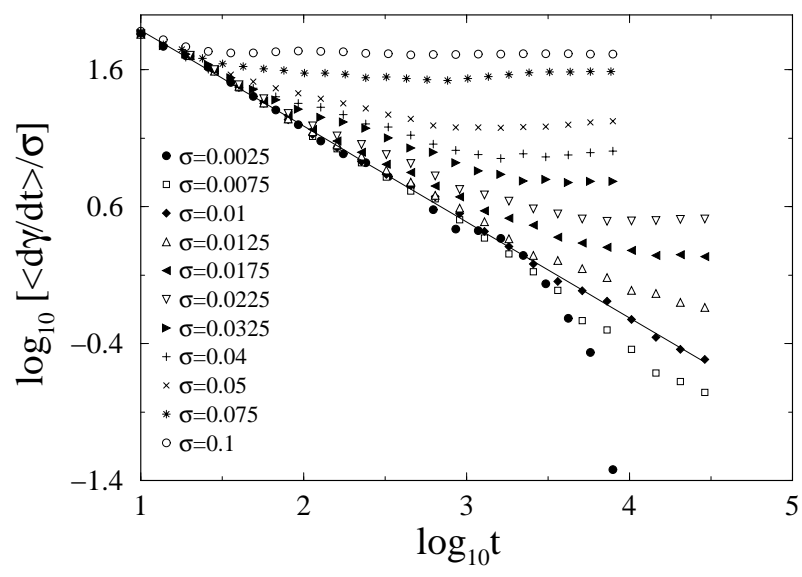

Figure 6 . The strain rate relaxation for different applied stresses at $T=0$ for a system of size $L=100 b$. The initial density of edge dislocations is around $1 \%$. The solid line is the best linear fit of the $\sigma=0.01$ curve and yields $d \gamma / d t \sim t^{-0.69}$.

These results suggested that a possible interpretation of dislocation motion and the corresponding creep laws of crystalline materials could also be found within the general "jamming" framework proposed to encompass the wide variety of non-equilibrium soft and glassy materials discussed previously. When jammed, these systems are unable to explore phase space, but they can be unjammed by changing the stress, the density, or the temperature. The analogies of dislocation motion and these so-called jammed systems was further explored by considering the influences of dislocation multiplication, and thermal-like fluctuations on the dynamics. Dislocation multiplication favors the rearrangements of the system and induces a linear creep regime (flowing phase) at lower stress values, but it does not affect the initial powerlaw creep. The introduction of a finite effective temperature $T$ had a similar effect [91]. 


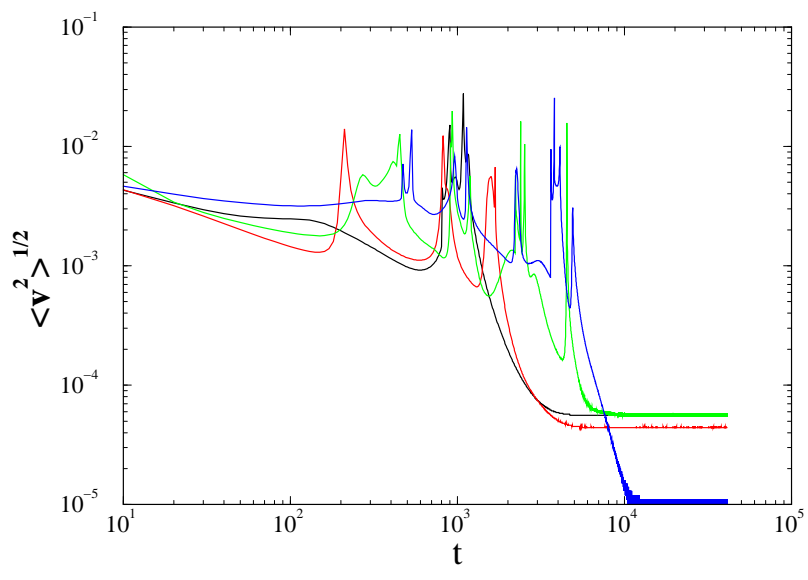

Figure 7. The time evolution of the root-mean-square velocity of four individual runs of the numerical simulations for different initial conditions. The applied stress value in all cases is $\sigma=0.0075$. The curves are depicted in a double logarithmic scale to emphasize the intermittent bursts characteristic of the creep dislocation dynamics around the yield threshold.

The detailed analysis of the model data unveiled the dislocation microscopic dynamics in the Andrade, and in the stationary regimes: Most dislocations are arranged into metastable structures so that the stress field they generate in the material is screened out on large length-scales. These structures consist of small-angle dislocation boundaries separating slightly misoriented crystalline blocks or far more complex dislocation arrangements. If the applied stress is below the yield threshold, dislocations are not able to easily explore the space of configurations to find the most favorable spatial arrangement and they are, most of the time, trapped in metastable configurations which induce a jamming of the system. Around the yield threshold, a small fraction of dislocations may, however, attain a higher mobility and provoke several intermittent rearrangements of the whole system in the course of time. The stress field generated by these unsettled dislocations conserves the initial long-range character, and forces the system to continue evolving in time in a cooperative manner to try to reduce the internal shear stress (or minimize the elastic energy) by exploring further more favorable arrangements.

In Fig. 7, we show the root-mean-square velocity $\left\langle v^{2}\right\rangle^{1 / 2}(t)=\left[\sum_{i} v_{i}^{2} / N\right]^{1 / 2}$ of all the dislocations $(N \sim$ $100-150)$ present in a square cell of size $L=100 \mathrm{~b}$ as a function of time for four single runs of the numerical simulations. Thus, each run represents the creep behavior of a small piece (a few nanometers big) of a macroscopic system, and starts from a different initial dislocation configuration, obtained after letting the system relax in the absence of external load during a given time interval. The external shear stress applied is in all cases $\sigma=0.0075$, that is, in the vicinity of the critical threshold $\sigma_{c}$. We can clearly appreciate the presence of a few intermittent burst after which $\left\langle v^{2}\right\rangle^{1 / 2}(t)$ slowly decreases in time. Similar burst, but either positive or negative, can also be observed in the corresponding strainrate curves $d \gamma / d t$ (not shown). Andrade's power law creep appears as a result of the averaging process over many of these runs, mimicking the behavior of a much bigger system. The closer is the applied stress to the threshold the longer is the collective power-law motion before the system falls either in the jammed or in the moving state. Precisely at the critical point and for the case of an infinite system, the Andrade power-law could in principle last indefinitely.

\section{Non-linear rheology}

Above the stress threshold, the system eventually exhibits a linear creep regime in which the dislocations present in the system tend to glide in a coherent manner. The dependence of the steady strain-rate value on the external shear stress is shown in Fig. 8. Within the error bars, the simulation data for the higher stress values considered can be fit quite nicely by a cubic law dependence (see the solid line in the plot). This is an interesting result since, if we were to compare with the nonlinear rheology characteristic of amorphous polymeric networks or other soft glassy materials [106], it would correspond to an effective shear-thinning viscosity for the dislocation ensemble which decreases with the strain-rate as $\eta=\sigma(d \gamma / d t)^{-1} \sim(d \gamma / d t)^{-2 / 3}$. This result is in good agreement with the theoretical results obtained in Ref. [106] and compatible with the power law shear-thinning behavior $\eta \sim \dot{\gamma}^{-\alpha}$ with $\alpha=0.5-1.0$ observed in many different complex fluids [7].

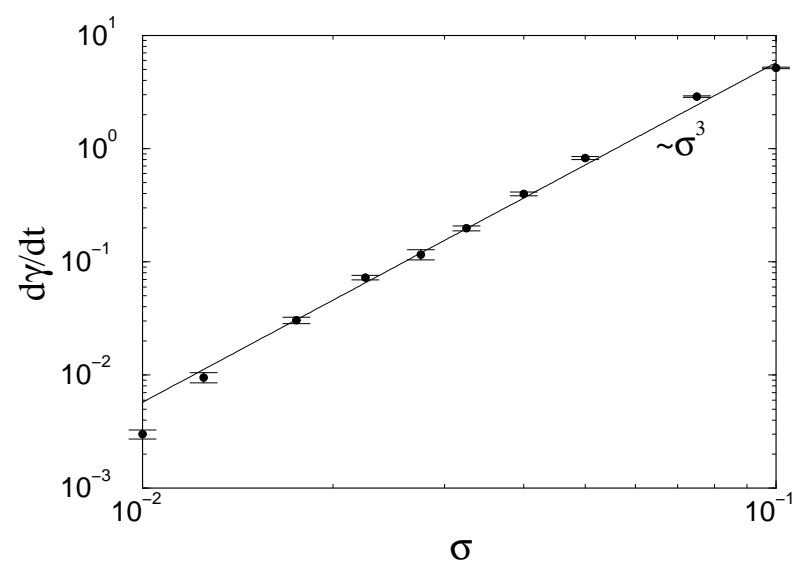

Figure 8 . The steady strain rate value for different applied stresses in a double logarithmic scale. The solid line represents a cubic dependence of the form $d \gamma / d t \sim \sigma^{3}$ which appears to be in good correspondence with the simulation data for the higher stress values considered.

To summarize, the flow of dislocation structures in crystalline solids undergoing plastic deformation shares common features with the time-dependent linear rheology and with the nonlinear steady rheology of soft glassy materials and, in particular, it seems to satisfy the empirical Cox-Merz rule. Notice, however, that the concentration of dislocations in the crystal needs not to be too high to warrant the presence of kinematical constraints and metastability in the dynamics. High concentrations could be replaced in this case by the long-range character of their mutual elastic interactions, that favor collective motions and rearrangements, and by their ability to form intricate extended spatial structures (in order to screen out the stress), that tend to glide in a coherent manner and thus can hamper their own relative motions driving the system to a jammed state. Further work is currently under way to try to precisely identify the most basic mechanism responsible for these remarkable similarities. 


\section{Conclusions}

In this paper we have discussed the collective dynamics of an assembly of interacting particles and, in particular, the transition from a blocked to a moving phase. Transitions of this kind are observed in different contexts and are due to different mechanisms. When the particles are blocked by quenched disorder, one typically refers to the depinning transition, which can be elastic when the medium preserves its topology through the transition, or plastic when topological defects, such as dislocations, are generated during the dynamics. The driving force for depinning can be due to an externally applied field, or could be self-generated by a density gradient, as in the case of front propagation. When the motion is not hindered by quenched disorder, but by intrinsic constraints one usually refers to a jamming transition.

Common features of depinning and jamming phenomena are, at the macroscopic level, the observation of a nontrivial steady-state force-velocity curve, scaling typically as $v \sim\left(F-F_{c}\right)^{\beta}$ for $F>F_{c}$, and a transient power law relaxation of the velocity $v \sim t^{-\alpha}$. At the microscopic level, pinning and jamming systems are both characterized by a complex energy landscape, with many metastable states. This leads to an intermittent avalanche-like response to external perturbations. Thus despite the different origins, pinning and jamming have several properties in common which could be possibly used to construct a comprehensive theory of deblocking transitions. Numerical simulations of interacting particles have played a major role so far to elucidate the detailed nature of some of these phenomena. The advancement of theoretical understanding is needed to redirect numerical simulations from a purely descriptive point of view to a deeper level of analysis.

\section{Acknowledgements}

We thank A. A. Moreira, J. Mendes-Filho, A. Vespignani and M. Zaiser, who have contributed to the work reviewed here. We thank H. F. da Silva for the numerical results reported in Fig. 4. This work is supported by an ItalySpain Integrated Action. MCM is supported by the Ministerio de Ciencia y Tecnología (Spain). JSA is supported by CNPq. SZ acknowledges FUNCAP for supporting his visit to the Universidade Federal do Ceará.

\section{References}

[1] C. Murray, C. Kagan, and M. Bawendi, Ann. Rev. Mat. Sci. 30, 545 (2000).

[2] T. B. Mitchell, J. J. Bollinger, W. M. Itano, and D. H. E. Dubin, Phys. Rev. Lett. 87, art n. 183001 (2001).

[3] A. Pertsinidis and X. S. Ling, Nature (London) 413, 404 (2001).

[4] J. Marro and R. Dickman, Nonequilibrium Phase Transitions in Lattice Models (Cambridge University Press, Cambridge, 1999).

[5] H. Hinrichsen, Braz. J. Phys. 30, 69 (2000); J. F. F. Mendes, Braz. J. Phys. 30, 105 (2000); H. Park and S. Kwon, Braz. J. Phys. 30, 133 (2000).
[6] D. Rapaport, The Art of Molecular Dynamics Simulation (Cambridge University Press, Cambridge, 1995).

[7] R.G. Larson, The structure and rheology of complex fluids, (Oxford University Press, New York, 1999).

[8] J.F. Brady and G. Bossis, Annu. Rev. Fluid Mech. 20, 111 (1988).

[9] Y. Yamane, Y. Kaneda, and M. Doi, J. Non-Newtonian Fluid Mech. 54, 405 (1994).

[10] T. Ohta, H. Nozaki, and M. Doi, J. Chem. Phys. 93, 2664 (1990).

[11] S.A. Kahn and R.C. Armstrong, J. Non-Newtonian Fluid Mech. 22, 1 (1986)

[12] J.P. Hirth and J. Lothe, Theory of Dislocations (Krieger Publishing Company, 1992).

[13] M. Kardar, G. Parisi, and Y.-C Zhang, Phys. Rev. Lett. 56, 889 (1986).

[14] A.-L. Barabási and H. E. Stanley, Fractals Concepts in Surface Growth, (Cambridge University Press, Cambridge, 1995).

[15] T. Halpin Healy and Y.-C Zhang, Phys. Rep. 254, 215 (1995).

[16] S. He, G. L. M. K. S. Kahanda, and P-Z. Wong, Phys. Rev. Lett. 69, 3731 (1992).

[17] M. A. Rubio, C. A. Edwards, A. Dougherty, and J. P. Gollub, Phys. Rev. Lett. 63, 1685 (1989).

[18] J. P. Stokes, A. P. Kushnick, and M. O. Robbins, Phys. Rev. Lett. 60, 1386 (1988).

[19] M. Dube, M. Rost and M. Alava, Eur. Phys. J. B 15, 691 (2000).

[20] J. Zhang, Y.-C., Zhang, P. Alstrom, and M. T. Levinsen, Physica A 189, 383 (1992)

[21] J. Maunuksela, M. Myllys, O.-P. Kahkonen, J. Timonen, N. Provatas, M.J. Alava and T. Ala-Nissila, Phys. Rev. Lett. 79, 1515 (1997).

[22] A. Brú, J.M. Pastor, I. Fernaud, I. Brú, S. Melle, and C. Berenguer, Phys. Rev. Lett. 81, 4008 (1998).

[23] J. S. Andrade Jr., D. Street, Y. Shibusa, S. Havlin, and H. E. Stanley, Phys Rev. E 55, 772 (1997).

[24] M. Sahimi, Flow and Transport in Porous Media and Fractured Rock (VCH, Boston, 1995).

[25] S. Havlin and D. Ben-Avraham, Adv. Phys. 36, 695 (1987).

[26] S. Havlin and A. Bunde, "Percolation II: Transport in Percolation Clusters" in Fractals and Disordered Systems, Second Edition edited by A. Bunde and S. Havlin (Springer-Verlag, Berlin, 1996)

[27] E. H. Brandt, Rep. Prog. Phys. 58, 1465 (1995).

[28] G. Blatter, M.V. Feigel'man, V.B. Geshkenbein, A.I. Larkin, and V.M. Vinokur, Rev. Mod. Phys. 66, 1125 (1994).

[29] C. P. Bean, Rev. Mod. Phys. 36, 31 (1964).

[30] Y. B. Kim, C. F. Hempstead and A. R. Strnad, Phys. Rev. 129, 528 (1963).

[31] S. Field, J. Witt, F. Nori, and X. Ling, Phys. Rev. Lett. 74, 1206 (1995); C. M. Argenter, Phys. Rev. B 58, 1438 (1998); K. Behnia, C. Capan, D. Mailly and B. Etienne, Phys. Rev. B 61, R3815 (2000). 
[32] R. Surdeanu, R. J. Wijngaarden, E. Visser, J. M. Huijbregtse, J. H. Rector, B. Dam, and R. Griessen, Phys. Rev. Lett. 83, 2054 (1999).

[33] R. Surdeanu, R. J. Wijngaarden, B. Dam, J. Rector, R. Griessen, C. Rossel, Z. F. Ren and J. H. Wang, Phys. Rev. B 58, 12467 (1998).

[34] S. S. James, S. B. Field, J. Seigel and H. Shtrikman, Physica C 332, 445 (2000).

[35] H.J. Jensen, A. Brass and A.J. Berlinsky, Phys. Rev. Lett. 60, 1676 (1988).

[36] O. Pla and F. Nori, Phys. Rev. Lett. 67, 919 (1991); R. Richardson, O. Pla, and F. Nori, Phys. Rev. Lett. 72, 1268 (1994); W. Barford, W. H. Beere and M. Steer, J. Phys.: Condens. Matter 5, L333 (1993).

[37] C. Reichhardt, J. Groth, C.J. Olson, S. Field, and F. Nori, Phys. Rev. B 52, 10441 (1995); 53, R8898 (1996).

[38] C. Reichhardt, C.J. Olson, J. Groth, S. Field, and F. Nori, Phys. Rev. B 54, 16108 (1996); 56, 14196 (1997).

[39] O. Pla, N. K. Wilkin and H. J. Jensen, Europhys. Lett. 33, 297 (1996).

[40] C.J. Olson, C. Reichhardt, and F. Nori, Phys. Rev. B 56, 6175 (1997).

[41] W. Barford, Phys. Rev. B 56, 425 (1997).

[42] K. E. Bassler and M. Paczuski, Phys. Rev. Lett. 81, 3761 (1998); G. Mohler and D. Stroud, Phys. Rev. B 60, 9738 (1999).

[43] D. Monier and L. Fruchter, Eur. Phys. J. B. 17, 201 (2000).

[44] C. Caroli and P. Nozieres, Eur. Phys. J. B. 4, 23 (1998).

[45] S. Zapperi, A. A. Moreira and J. S. Andrade Jr., Phys. Rev. Lett. 86, 3622 (2001).

[46] A. A. Moreira, J. S. Andrade Jr., Mendes Filho J., and S. Zapperi, Phys. Rev. B 66, 174507 (2002).

[47] V. V. Bryskin and S. N. Dorogotsev, JEPT 77, 791 (1993); Physica C 215, 173 (1993).

[48] J. Gilchrist and C. J. van der Beek, Physica C 231, 147 (1994).

[49] G. Grüner, A. Zawadowski and P. M. Chaikin, Phys. Rev. Lett. 46, 511 (1981).

[50] D. S. Fisher, Phys. Rev. B 31, 1396 (1985).

[51] S. Zapperi, J. S. Andrade and J. Mendes Filho, Phys. Rev. B 61, 14791 (2000).

[52] M. Kardar, Phys. Rep. 301, 85 (1998).

[53] T. Nattermann, S. Stepanow, L. H. Tang, and H. Leschhorn, J. Phys. II (France) 2, 1483 (1992).

[54] O. Narayan and D. S. Fisher, Phys. Rev. B 48, 7030 (1993).

[55] H. Leschhorn, T. Nattermann, S. Stepanow, and L. H. Tang, Ann. Physik 6, 1 (1997).

[56] D. Ertas and M. Kardar, Phys. Rev. E 49, R2532 (1994).

[57] P. Chauve, T. Giamarchi, and P. Le Doussal, Phys. Rev. B 62, 6241 (2000).

[58] P. Le Doussal, K. J. Wiese, and P. Chauve, Phys. Rev. B66, 174201 (2002).
[59] N. W. Ashcroft and N. D. Mermin, Solid State Physics, (Holt, Rinehart and Winston, Philadelfia, 1976).

[60] T. Giamarchi and P. Le Doussal, Phys. Rev. Lett. 76, 3408 (1996); Phys. Rev. Lett. 78, 752 (1997); Phys. Rev. B 57, 11356 (1998).

[61] L. Balents, M. C. Marchetti, and L. Radzihovsky, Phys. Rev. Lett. 78, 751 (1997).

[62] A. I. Larkin and Yu. N. Ovchinnikov, J. Low Temp. Phys. 34, 409 (1979).

[63] D. Cule and T. Hwa, Phys. Rev. Lett. 77, 278 (1996).

[64] D. Cule and T. Hwa, Phys. Rev. B 57, 8235 (1998).

[65] F. Lacombe, S. Zapperi, and H. J. Herrmann, Phys. Rev. B 63, 104104 (2001).

[66] C. Reichhardt and C. J. Olson, Phys. Rev. Lett. 89, 078301 (2002).

[67] M. C. Faleski, M. C. Marchetti, and A. A. Middleton, Phys. Rev. B54, 12427 (1996).

[68] M. C. Marchetti, A. A. Middleton, and T. Prellberg, Phys. Rev. Lett. 85, 1104 (2000).

[69] M. C. Marchetti and K. A. Dahmen, Phys. Rev. B66, 214201 (2002).

[70] B. N. J. Persson, Sliding Friction (Springer, Berlin, 1998).

[71] C. J. Olson, C. Reichhardt, and F. Nori, Phys. Rev. Lett. 80, 2197 (1998).

[72] H. Fangohr, S. J. Cox, and P. A. J. de Groot, Phys. Rev. B64, 064505 (2001).

[73] J. Watson and D. S. Fisher, Phys. Rev. B 54, 938 (1996).

[74] J. Watson and D. S. Fisher, Phys. Rev. B55, 14909 (1997).

[75] T. Kawaguchi, Phys. Stat. Sol.213, R3 (1999); Phys. Lett. A251, 73 (1999).

[76] P. Bak, C. Tang, and K. Wiesenfeld, Phys. Rev. Lett.59, 381 (1987).

[77] R. Dickman, M. A. Muñoz, A. Vespignani, and S. Zapperi, Braz. J. Phys. 30, 27 (2000).

[78] S. S. Manna, J. Phys. A24, L363 (1991).

[79] R. Pastor-Satorras and A. Vespignani, Phys. Rev. E62, 6195 (2000).

[80] M. Paczuski and K.E. Bassler, Phys. Rev. E 62, 5347 (2000).

[81] M. Kloster, S. Maslov, and C. Tang, Phys. Rev. E 63, 026111 (2001).

[82] T. Giamarchi and S. Bhattacharya, Lecture notes of the 2001 Cargese school on "Trends in high magnetic field science", cond-mat/0111052.

[83] A. E. Koshelev and V. M. Vinokur, Phys. Rev. Lett. 73, 3580 (1994).

[84] C. J. Olson, C. Reichhardt, and F. Nori, Phys. Rev. Lett.81, 3757 (1998).

[85] I. F. Lyuksyutov, T. Nattermann, and V. L. Pokrovsky, Phys. Rev. B 59, 4260 (1999).

[86] S. Zapperi, G. Durin, A. Magni, and S. Zapperi, J. Magn. Magn. Mat. 242-245P2, 987 (2002).

[87] L. Bocquet, H. J. Jensen, J. Phys. I (France) 7, 1603 (1997).

[88] A. Glatz, T. Nattermann, and V. Pokrovsky, Phys. Rev. Lett.90, 047201 (2003). 
[89] A.J. Liu and S.R. Nagel, Nature 396, 21 (1998).

[90] A.J. Liu and S.R. Nagel (Eds.), Jamming and Rheology, (Taylor and Francis, London, 2001).

[91] M.-C. Miguel, A. Vespignani, M. Zaiser and S. Zapperi, Phys. Rev. Lett. 89, 165501 (2002)

[92] D. Bonn, S. Tanase, B. Abou, H. Tanaka, and J. Meunier, Phys. Rev. Lett. 89, 015701 (2002).

[93] T.G. Mason, J. Bibette, and D.A. Weitz, J. Colloid Interface Sci. 179, 439 (1996).

[94] D.J. Durian, D.J. Pine, and D.A. Weitz, Science 252, 686 (1991)

[95] P.N. Segrè, V. Prasad, A.B. Schofield, and D.A. Weitz, Phys. Rev. Lett. 86, 6042 (2001).

[96] H. Bissig, V. Trappe, S. Romer, and Luca Cipelletti, condmat/0301265.

[97] M. Cloitre, R. Borrega, F. Monti, and L. Leibler, Phys. Rev. Lett. 90, 068303 (2003).

[98] B. Fabry et al., Phys. Rev. Lett. 87, 148102 (2001).

[99] J.D. Ferry, Viscoelastic Properties of Polymers (Wiley, New York, 1980), 3rd ed.

[100] D.J. Plazek, X.D. Zheng, and K.L. Ngai, Macromolecules 25, 4920 (1992).

[101] H.A. Barnes, J.F. Hutton, and K. Walters, An Introduction to Rheology (Elsevier, Amsterdam, 1989).

[102] S.M. Fielding, P. Sollich, and M.E. Cates, J. Rheol. 44, 323 (2000)

[103] P. Sollich, F. Lequeux, P. Hébraud, and M.E. Cates, Phys. Rev. Lett. 78, 2020 (1997).
[104] M.L. Falk and J.S. Langer, Phys. Rev. E 57, 7192 (1998).

[105] J.-L. Barrat and L. Berthier, Phys. Rev. E 63, 012503 (2001).

[106] L. Berthier and J.-L. Barrat, J. Chem. Phys. 116, 6228 (2002).

[107] J. Rottler and M.O. Robbins, Phys. Rev. Lett. 89, 195501 (2002).

[108] W.K. Kegel and A. van Blaaderen, Science 287, 290 (2000).

[109] E. Weeks et al., Science 287, 627 (2000).

[110] W.P. Cox and E.H. Merz, J. Polym. Sci. 28, 619 (1958).

[111] M. Renardy, J. Non-Newtonian Fluid Mech. 68, 133 (1997).

[112] L. Berthier private communication (2002).

[113] J. Friedel, Dislocations (Pergamon Press, Oxford, 1967).

[114] A. H. Cottrell, Dislocations and Plastic Flow in Crystals (Oxford University Press, London, 1953).

[115] F.R.N. Nabarro, Theory of Crystal Dislocations (Dover, New York, 1992).

[116] E.N. da C. Andrade, Proc. R. Soc. London A 84, 1 (1910); 90, 329 (1914).

[117] N.F. Mott, Phil. Mag. 44, 741 (1953).

[118] A.H. Cottrell, Phil. Mag. Lett. 73, 35 (1996); 74, 375 (1996); 75, 301 (1997).

[119] F.R.N. Nabarro, Phil. Mag. Lett. 75, 227 (1997).

[120] M.-C. Miguel, A. Vespignani, S. Zapperi, J. Weiss and J. R. Grasso, Nature 410, 667 (2001). 